\section{Revista Chilena \\ de Pediatría}

www.revistachilenadepediatria.cl

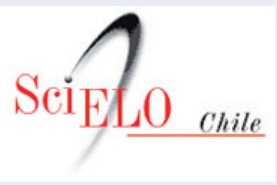

www.scielo.cl

\title{
Puertas Adentro: La otra cara de la pandemia
}

\section{Home confinement: the other side of the epidemic}

\author{
Marcela Abufhele ${ }^{\mathrm{a}}$, Valerie Jeanneret ${ }^{\mathrm{a}}$
}

aPsiquiatra Infantil y de la Adolescencia, Departamento Pediatría, Clínica Alemana de Santiago. Santiago, Chile
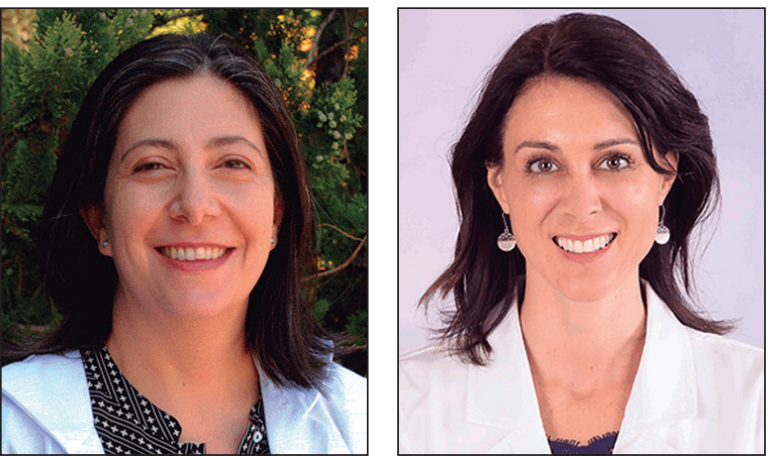

Estamos siendo golpeados por la mayor crisis sanitaria de los últimos 100 años. Esto ha obligado a los gobiernos de muchos países a establecer medidas para minimizar el contagio entre personas. Una de las medidas mas utilizadas para este propósito es la cuarente$n a$, es decir, la separación y restricción del movimiento de personas que potencialmente han estado expuestas a una enfermedad contagiosa para reducir el riesgo potencial de que infecten a otros ${ }^{1}$.

Nuestro país no ha sido la excepción. Desde el 25 de marzo, en que se inició la primera cuarentena masiva en territorio chileno hasta el día de hoy, se ha decretado cuarentena para 24 comunas y territorios, obligando a un total de 4.104 .900 personas a permanecer en sus casas (www.gob.cl/coronavirus).

La cuarentena, constituye una medida necesaria y efectiva para prevenir el contagio, y como profesionales de salud, debemos ser los primeros en fomentar su cumplimiento cuando la autoridad sanitaria así lo indica. Sin embargo, también debemos ser capaces de reconocer que la cuarentena altera en forma significativa la vida de las personas que deben realizarla y de la comunidad en general ${ }^{1}$. Son múltiples las formas en las cuales esta medida puede afectar el bienestar de niños, niñas, adolescentes y sus familias, independiente de sus indudables beneficios en "aplanar" la curva.

Según cifras de la Unesco ${ }^{2}$, el cierre de colegios y universidades alcanzó a más del 91\% de los estudiantes del mundo, esto es, 1570 millones de niños, niñas y jóvenes. En Chile, además de las medidas de cuarentena, todos los establecimientos educacionales se encuentran cerrados desde el 15 de marzo, interrumpiendo la rutina, el apoyo social de niños y adolescentes, además de representar factores de estrés adicionales para padres y madres, en condiciones de disminución y ausencia de sistemas habituales de cuidado infantil. Todo esto, aumenta significativamente la sobrecarga que provoca el confinamiento en casa y puede tener consecuencias de salud, tanto física como mental, e incluso más, alterar el desarrollo integral de la población infanto-adolescente y sus familias tanto a corto como a largo plazo ${ }^{1,3-5}$.

El documento técnico de la Alianza para la Protección de la Infancia en Acción Humanitaria ${ }^{5}$ sintetiza los riesgos de maltrato físico y emocional infantil en cuatro categorías: 
1. Negligencia y disminución de los cuidados de niños y adolescentes. Secundario a múltiples causas: cierre de escuelas y servicios de cuidado infantil; demandas laborales y personales constantes para los cuidadores, enfermedad, cuarentena o aislamiento de cuidadores.

2. Aumento del abuso y violencia doméstica e interpersonal, ya sea por aumento del estrés psicosocial de los cuidadores, o por ausencia de supervisión, aumento de tiempo de exposición a pantallas y contenidos de riesgos.

3. Aumento riesgo de lesiones, intoxicación y otros riesgos, secundarios a la mayor disponibilidad y uso indebido de desinfectantes tóxicos, alcohol y otros medicamentos.

4. Disminución del acceso a redes de apoyo protectoras intra o extrafamiliares: mayor aislamiento e invisibilidad de familias maltratadoras,y mayores dificultades para denunciar incidentes.

Estos riesgos a la protección de niños y jóvenes, tal como han sido señalados por UNICEF, surgen de lo observado en la actual pandemia del COVID-19 y de observaciones de brotes previos de enfermedades infecciosas. Son el resultado de medidas de control contra la propagación de enfermedades que no tienen en cuenta a las poblaciones vulnerables y los mecanismos de supervivencia negativos que éstas generan ${ }^{5}$.

Para nuestra población y contexto, esto se traduce en que una de las consecuencias más graves de esta pandemia "puertas adentro", es un aumento del riesgo de maltrato físico, psicológico y negligencia al interior de las familias ${ }^{5}$. Los miembros de la familia pasan la totalidad del tiempo confinados en la casa. Por un lado, los padres están angustiados y sobrepasados entre el cuidado de los hijos, labores del hogar, poder cumplir con el teletrabajo y la creciente preocupación por mantener el ingreso económico mínimo requerido. Por otro lado, están los niños y adolescentes sin asistencia escolar, sin poder ver a sus amigos ni participar en actividades de interés, con limitación de las vías habituales de liberación de tensión y energía, y aún sin clara adaptación a sistemas de educación en domici$\mathrm{lio}^{3}$. Lo anterior, puede generar aumento en niveles de irritabilidad, hiperactividad, impulsividad, y ansiedad que se agregan y agravan las múltiples tensiones a las que ya están sometidos los padres o cuidadores. Esta conjunción de factores puede tener un efecto de "olla a presión" y precipitar accidentes, respuestas impulsivas y/o violentas en un contexto donde las medidas protectoras de control externo están disminuidas ${ }^{3-5}$.

En un segundo nivel -pero no menos importantese encuentra un aumento de los riesgos de accidentes, intoxicaciones y abusos secundario a negligencia, disminución de la supervisión y los cuidados sobre los niños y adolescentes. En niños menores, puede haber un aumento de accidentes domésticos, derivado de la mayor hiperactividad y "aburrimiento" en un contexto de menor supervisión parental. Las intoxicaciones accidentales se hacen más frecuentes, dado que, a lo anterior, se suma la mayor disponibilidad de desinfectantes tóxicos, alcohol y otros medicamentos.

En el caso de adolescentes, el aburrimiento y búsqueda de novedad en este contexto lleva a mayor tiempo de exposición a pantallas y redes sociales sin supervisión de contenidos o contactos peligroso de posibles abusadores (cyberbulling, sexting, grooming) ${ }^{4,5}$.

Finalmente, se encuentran una amplia variedad de riesgos asociados al estrés psicosocial y trastornos de salud física y mental secundarios al confinamiento.

El cierre prolongado de los establecimientos educacionales y el confinamiento en casa afecta la salud física de los niños ${ }^{4}$. La evidencia sugiere que cuando los niños no están con clases presenciales realizan menor actividad física, tienen mayor tiempo de exposición a pantallas, y dietas menos saludables lo que se traduce en trastornos de sueño, aumento de peso y disminución de la capacidad funcional ${ }^{4,6}$. Una revisión del $2017^{6}$, demostró que la rutina diaria escolar estructurada es un factor protector para la regulación de comportamientos obesogénicos en la población infanto-juvenil.

El impacto psicólogico de la población infantoadolescente durante la cuarentena depende de varios factores: su duración, de cuánto miedo tengan de infectarse, que tan frustrados, aburridos y mal informados estén, el estrés económico familiar, el no tener suministros adecuados ni espacio personal en casa y la falta de contacto con compañeros y profesores del colegio ${ }^{4}$. También se describen estresores post cuarentena tales como las dificultades económicas en la familia y el estigma de haber estado enfermo o en contacto directo ${ }^{4}$.

La angustia generada por la enfermedad o muerte de seres queridos, síndrome de estrés agudo y postraumático, insomnio, irritabilidad, disminución de la concentración, aumento de consumo de alcohol/ drogas y aparición de trastornos ansiosos o depresivos en niños y familias vulnerables se observan durante la cuarentena y hasta 3 años después ${ }^{3,4}$. Los síntomas mas frecuentemente descritos durante la pandemia SARS en Hong Kong fueron: Inestabilidad emocional, ánimo bajo (73\%), estrés o ansiedad, Irritabilidad (57\%), insomnio, síntomas de despersonalización y desrealización y agotamiento emocional7 ${ }^{7}$. Es importante recordar que en la población infantojuvenil la forma en que se expresa el conflicto va a variar según el estadio de desarrollo psicológico en que se encuentre cada individuo.

Sobre las consecuencias en el largo plazo, un estudio después de la pandemia de SARS en Canadá estimó que la incidencia del trastorno de estrés postraumático 
(TEPT) fue similar a la de los desastres naturales y el terrorismo $(28,9 \%)^{8}$.

Otro estudio comparó los síntomas de estrés postraumático en los padres y los niños que estuvieron en cuarentena con los que los que no, durante el brote de H1N1 en 2009 y encontraron que las puntuaciones medias de TEPT eran cuatro veces más altas en los niños que habían sido puestos en cuarentena (33\%) que en los que no. También fue importante el hallazgo que de los padres con TEPT, $86 \%$ de sus hijos lo presentaba también'.

Por último, sabemos que la sobrecarga que el confinamiento en casa puede provocar es muchísimo mayor en pacientes y familias con patología de salud mental previa. Hay evidencia que los desastres afectan desproporcionadamente a las poblaciones pobres y vulnerables, y los pacientes con enfermedad mental y familias disfuncionales pueden estar entre los más afectados ${ }^{9-11}$.

Sin duda, esta pandemia traerá consecuencias negativas y positivas que aún no dimensionamos en su totalidad. Como clínicos, entender estos riesgos y los mecanismos por los cuales se puede afectar la vida de los niños y adolescentes es crucial para anticipar, prevenir y derivar a nuestros pacientes y sus familias de manera de minimizar el potencial de consecuencias negativas en el presente y a futuro ${ }^{4,12}$.

En crisis, la atención centrada en la familia ha sido un sello distintivo de los profesionales que atendemos niños y debemos estar más activos que nunca en educar a los padres en tomar medidas de cuidado y protección no solo del contagio sino también del bienestar y de los posibles accidentes. No poner el foco solo en la exigencia y demanda académica sino también en el auto y heterocuidado. En la evaluación clínica, estar atento a signos de alarma de malos tratos y afectación emocional significativa, junto con transmitir la importancia de cuidar, dentro de lo posible, las rutinas de sueño, alimentación, actividad física y tiempo de exposición a pantallas ${ }^{4,5,12}$.

Sin duda que las crisis, constituyen también una oportunidad, y como tal, podemos aprovechar esta pandemia y darle un sentido positivo y esperanzador. Pocas veces en la historia han existido momentos en que las familias se ven forzadas a volcarse "puertas adentro", y, surge entonces, una oportunidad de reencontrarse en las cenas compartidas, en las labores del hogar, en el disfrute de jugar juntos o en las conversaciones sin prisa; y de fomentar en nuestros niños la autonomía, responsabilidad, creatividad y empatía.

\section{Conflicto de intereses:}

Las autoras declaran no tener conflicto de intereses.

\section{Referencias}

1. Brooks SK, Webster RK, Smith LE, et al. The psychological impact of quarantine and how to reduce it: rapid review of the evidence. Lancet 2020; published online Feb 19. https://doi.org/10.1016/ S22150366(20)30077-8.

2. UNESCO, 2020. Coalición Mundial para la Educación COVID-19. https://es.unesco.org/covid19/ globaleducationcoalition

3. UNESCO, 2020. COVID-19 educational disruption and response. https:// en.unesco.org/themes/educationemergencies/coronavirus-schoolclosures

4. Wang G, Zhang Y, Zhao J, Zhang J, Jiang F. Mitigate the effects of home confinement on children during the COVID-19 outbreak. The Lancet 2020;395(10228):945-7. https://doi. org/10.1016/s0140-6736(20)30547-x
5. Alliance for Child Protection in Humanitarian Action. Protection of children during the coronavirus disease (COVID-19) pandemic: Technical note. https://www.unicef.org/documents/ technical-note-protection-childrencoronavirus-disease-2019-covid-19pandemic

6. Brazendale K, Beets MW, Weaver RG, et al. Understanding differences between summer vs. school obesogenic behaviors of children: the structured days hypothesis. Int J Behav Nutr Phys Act 2017;14:100.

7. Lee S, Chan LY, Chau AM, Kwok KP, Kleinman A. The experience of SARSrelated stigma at Amoy Gardens. Soc Sci Med 2005;61:2038-46.

8. Hawryluck L, Gold WL, Robinson S, Pogorski S, Galea S, Styra R. SARS control and psychological effects of quarantine, Toronto, Canada. Emerg Infect Dis. 2004;10(7):1206-121.
9. Sprang G, Silman M. Posttraumatic Stress Disorder in Parents and Youth After Health-Related Disasters. Disaster Medicine and Public Health Preparedness 2013;7(1):105-10. https://doi.org/10.1017/dmp.2013.22

10. Reynolds DL, Garay JR, Deamond SL, Moran MK, Gold W, Styra R. Understanding, compliance and psychological impact of the SARS quarantine experience. Epidemiol Infect 2008; 136: 997-1007.

11. Druss BG. Addressing the COVID-19 Pandemic in Populations With Serious Mental Illness. JAMA Psychiatry 2020. https://doi.org/10.1001/ jamapsychiatry.2020.0894

12. Liu JJ, Bao Y, Huang X, Shi J, Lu L. Mental health considerations for children quarantined because of COVID-19. The Lancet Child \& Adolescent Health 2020;4(5):347-9. 